\title{
PREDADORES ASOCIADOS A Panonychus citri McGREGOR (ACARI: TETRANYCHIDAE) EN LA COSTA CENTRAL DEL PERÚ
}

\section{PREDATORS ASSOCIATED TO Panonychus citri McGREGOR (ACARI: TETRANYCHIDAE) IN THE CENTRAL COAST OF PERU}

\author{
Alberto Daniel Guanilo y Norberta Martínez ${ }^{2}$
}

\section{Resumen}

Fueron identificadas 15 especies de predadores asociados a Panonychus citri en la costa central del Perú. Estos fueron: Amblyseius chungas Denmark \& Muma, Phytoseiulus persimilis AthiasHenriot, Neoseiulus californicus (McGregor), Typhlodromus (Anthoseius) evectus (Schuster), Typhlodromus (Anthoseius) transvaalensis (Nesbitt), Typhlodromina subtropica Muma \& Denmark, Mexecheles sp., Cheletogenes sp. y Armascirus sp. Los insectos predadores fueron Stethorus tridens Gordon, Stethorus histrio Chazeau, Oligota sp., Chrysoperla externa Hagen, Ceraeochrysa cincta Schneider y Aeolothrips sp. Las especies predadoras asociadas más frecuentes fueron A. chungas, T. (A.) evectus y N. californicus. Asimismo, Stethorus tridens y Stethorus histrio fueron abundantes en zonas donde no se encontraron los ácaros predadores antes mencionados.

Palabras claves: Panonychus citri, Amblyseius chungas, Neoseiulus californicus, Typhlodromus evectus, Perú

\begin{abstract}
In the central coast of Peru, fifteen predators associated to Panonychus citri were identified: Amblyseius chungas Denmark \& Muma, Phytoseiulus persimilis Athias-Henriot, Neoseiulus californicus (McGregor), Typhlodromus (Anthoseius) evectus (Schuster), Typhlodromus (Anthoseius) transvaalensis (Nesbitt), Typhlodromina subtropica Muma \& Denmark, Mexecheles sp., Cheletogenes sp. and Armascirus sp. Stethorus tridens Gordon, Stethorus histrio Chazeau, Oligota sp., Chrysoperla externa Hagen, Ceraeochrysa cincta Schneider and Aeolothrips sp. were the acarophagous insects found. Most abundant species were: A. chungas, T.(A.) evectus and $N$. californicus. Furthermore, both Stethorus tridens and Stethorus histrio were frequent in fields where predator mites were absent.
\end{abstract}

Key words: Panonychus citri, Amblyseius chungas, Neoseiulus californicus, Typhlodromus evectus, Peru

\section{Introducción}

"La arañita roja de los cítricos” Panonychus citri McGregor es uno de los ácaros más nocivos de cítricos en el Perú, según los resultados de la última evaluación de las principales plagas en cítricos a lo largo de la costa del Perú (ProCitrus, 2005).

En el Perú son escasas las investigaciones realizadas acerca de los ácaros fitoseidos como biocontroladores de $P$. citri. En los primeros estudios de $P$. citri en Perú fueron registrados a Stethorus sp. (Coccinellidae) (Beingolea, 1959), Somatium sp. (Staphylinidae) (Beingolea et al., 1968) y "ácaros predadores” (Beingolea et al., 1969), como controladores biológicos de P. citri. Posteriormente, Aguilar et al, (1980) registraron a los insectos predadores tales como Stethorus sp. (Coccinellidae) y Somatium oviformis (Staphylinidae) y a los ácaros predadores Amblyseius sp. y Pyemotes sp. Yauri (2001) realizó una colecta de ácaros controladores biológicos de $P$. citri, encontrando ácaros predadores pertenecientes a la familia Phytoseiidae.
En el presente trabajo, se estudian los ácaros e insectos predadores asociados a $P$. citri en los cultivos de cítricos de la costa central del Perú.

\section{Materiales y métodos \\ Reconocimiento de $\boldsymbol{P}$. citri y predadores asociados}

Las colectas de los controladores de P.citri se realizaron en 16 pomares de cítricos (Tabla 1) ubicados en los Departamentos de Lima (Irrigación Santa Rosa, Huaral y Cañete) e Ica (Chincha y Nazca). Las colectas fueron realizadas entre Octubre del 2003 y Abril del 2005. Cada muestreo fue realizado en un periodo de cinco horas diurnas, durante el cual se recorrió el campo de cultivo en zig-zag, colectándose hojas o frutos de las partes medias de las plantas que estuviesen infestadas con predadores de $P$. citri, con la ayuda de una lupa de 20X. Las hojas y frutos con $P$. citri y sus predadores se retiraron de la planta utilizando tijeras y fueron colocadas en bolsas plásticas de 3”x10” con alcohol al 70\% y se procedió a codificarlas. Luego, los ácaros predadores fueron 
montados según la metodología propuesta por Pritchard \& Baker (1955). Para reconocimiento de los ácaros predadores se usaron las claves de identificación de Krantz (1978). Las especies de ácaros Phytoseiidae se realizó por comparación con las descripciones originales de las especies o con las redescripciones de las mismas. La identificación se realizó con microscopio de contraste de fase. Quedaron depositados algunos ejemplares en el Museo de Historia Natural "Javier Prado" (LimaPerú) y en el Museo de Acarologia de la "Escola Superior de Agricultura Luiz de Queiroz” (Piracicaba, Sao Paulo - Brasil).

Los insectos predadores como crisopas (Neuroptera) y escarabajos (Coleoptera) fueron aclarados con $\mathrm{NaOH}$ al $10 \%$, luego lavado con agua destilada y se volvieron a colocar en alcohol de $70 \%$. El montaje fue temporal, usando láminas excavas con alcohol glicerado. Para thrips (Thysanoptera), se usó el montaje permanente. Las observaciones fueron realizadas con microscopio estereoscopio y microscopio de fase luminosa. Los neurópteros fueron identificados con la clave de Nuñez (1988), los coleópteros con la clave de Gordon \& Chapin (1983) y Navarrete-Heredia et al. (2002) y los thrips con la clave de Stehr (1991).

\section{Resultados}

Se colectaron un total de 2355 ejemplares de predadores adultos asociados a $P$. citri. Pertenecientes a quince especies, entre ácaros e insectos (Tabla 1). Éstas fueron: Amblyseius chungas, Typhlodromus (A.) evectus, Typhlodromus (A.) transvaalensis, Typhlodromina subtropica, Phytoseiulus persimilis, Neoseiulus californicus, Mexecheles sp., Cheletogenes sp., Armascirus sp., Stethorus tridens, Stethorus histrio, Ceraeochrysa cincta, Aeolothrips sp., Chrysoperla externa y Oligota sp.

Los ácaros predadores asociados a $P$. citri más frecuentes fueron: Amblyseius chungas, Typhlodromus (Anthoseius) evectus y Neoseiulus californicus.

Asimismo $S$. histrio y $S$. tridens fueron los insectos predadores más frecuentes y abundantes, especialmente en áreas donde no fueron encontraron los ácaros predadores antes mencionados. Las especies restantes se presentaron raramente.

Determinación de los predadores asociados a $\boldsymbol{P}$. citri

\section{Los ácaros predadores asociados a de $\boldsymbol{P}$. citri}

Se colectaron seis especies de la familia Phytoseiidae, dos especies de la familia Cheyletidae y una especie de la familia Cunaxidae.

\section{I.1. Familia Phytoseiidae Berlese 1916 Subfamilia: AMBLYSEIINAE Muma 1961}

\section{Género: Amblyseius Berlese 1914}

\section{Amblyseius chungas Denmark \& Muma 1989 (Figura 1)}

Material examinado: Fundo María Luisa (Huaral Lima): Citrus reticultata 20.X.3 (2), Fundo El Paraíso (Irrigación Santa Rosa - Lima): Citrus sinensis var. washington navel 24.X.3 (1), Fundo Hoja Redonda (Chincha - Ica) Citrus unshiu var. owari (3) 3.XI.3, Fundo Ramos (Cañete - Lima): M. owari x M. king y M. clementina x M. dancy (3) 27.XI.3, 8.I.4, 4.III.4, 1.IX.4, 31.III.5, 2.II.5, 7.IV.5, Fundo San Martín (Cañete - Lima): Citrus clementina 28.XI.3 (5), Fundo Tranquera Blanca (Cañete - Lima): M. clementina x M. dancy 23.XII.3 (3), Fundo El Rancho (Chincha -Ica) Citrus reticultata 20.I.4 (3), Fundo La Calera (Chincha-Ica): M. king x M. dancy 28.I.4 (2), Fundo Oscar Ramos (Cañete - Lima): Citrus reticultata 28.II.5 (1), Fundo Agropec (Cañete Lima) M. owari x M. king y Citrus unshiu (M.) var. owari 3.III.5 (2), Donoso INIA-Huaral (Huaral Lima): C. paradisi x C. reticulata 15.IV.5 (3), Fundo La Tapera (Huaral - Lima): Citrus unshiu var. owari 15.IV.5, 18.IV.5, 20.IV.5 (3).

Observaciones: Las medidas de 31 hembras adultas colectadas en este trabajo son semejantes a la descripción original, excepto que los especimenes colectados son más grandes que los reportados por Denmark \& Muma (1989) (Motupe - Perú), y también las setas son más largas, siendo muy notorio en la seta Z5. Además la espermateca es semejante en forma, pero es aproximadamente cuatro veces más larga que la descripción original. Los promedios y el intervalo (en paréntesis) de las medidas del cuerpo en $\mu \mathrm{m}$ son: la placa dorsal 354 (335-365) de largo y 212 (185245) de ancho; j1 31 (27-35), j3 59 (52-62), j4 7 (5-8), j5 6 (5-7), j6 7 (6-10), J2 8 (6-10), J5 9 (7-10), z2 12 (9-15), z4 13 (11-18), z5 6 (4-8), Z1 8 (6-10), Z4 136 (115-145), Z5 253 (240-288), s4 114 (110-130), S2 8 (7-11), S4 8 (5-10), S5 8 (6-11), r3 19 (14-22), R1 9 (7-12); Sge IV 112 (100-121), Sti IV 83 (70-93), St IV 69 (65-75); St1-St3 64 (62-68), St2-St2 77 (75-80), St5-St5 72 (70-76), la placa ventrianal 118 (100-130) de largo y 82 (65-95) de ancho; cáliz de la espermateca 22 (18-25) de largo. Peritrema presente desde la seta $\mathrm{j} 1$.

Se observó depredando a P.citri, tanto en laboratorio como en campo. Las posturas son colocadas separadas una de otra. Se observó que además de alimentarse de P.citri, se alimenta de otros ácaros plaga como Polyphagotarsonemus latus (Acari: Tarsonemidae). Esta especie también ha sido encontrada en Ricinus communis (Euphorbiaceae) en Cañete. 


\section{Género: Phytoseiulus Evans 1952 Phytoseiulus persimilis Athias-Henriot 1957}

(Figura 2)

Material examinado: Fundo La Calera (Chincha Ica): M. king x M. dancy 28.I.4 (4).

Observaciones: Las medidas de 4 hembras adultas colectadas en este trabajo son semejantes a la descripción de Denmark \& Schicha (1983), excepto que la seta r3 es cerca de un $40 \%$ más corta. Los promedios y el intervalo (en paréntesis) de las medidas del cuerpo en $\mu \mathrm{m}$ son: la placa dorsal 337 (325-345) de largo y 184 (180-190) de ancho; j1 25 (25-27), j3 47 (45-48), j4 55 (52-61), j5 72 (70-74), j6 142 (142-143), J5 6 (5-6), z2 14 (13-15), z4 59 (5862), z5 10(9-10), Z1 112 (110-115), Z4 130 (125125), Z5 128 (125-132), s4 167 (165-170), r3 14 (1315), R1 24 (21-29); Sge IV 75 (60-85), Sti IV 35 (3241), St IV 111 (110-113), la placa ventrianal 79 (7084) de largo y 68 (65-72) de ancho; cáliz de la espermateca 28 (25-30) de largo. Peritrema presente entre z2 y z4. Esta especie también ha sido encontrada en Ricinus communis (Euphorbiaceae) en Cañete.

\section{Género: Neoseiulus Hughes 1948}

\section{Neoseiulus californicus McGregor 1954 (Figura 3)}

Material examinado: Fundo María Luisa (Huaral Lima): Citrus reticultata 20.X.3 (2), Fundo Hoja Redonda (Chincha-Ica) Citrus unshiu var. owari (3) 3.XI.3, Fundo La Calera (Chincha - Ica): M. king x M. dancy 28.I.4 (3), Fundo Luis Calle (Irrigación Santa Rosa - Lima): Mandarina Citrus reticultata 15.II.5 (1), Fundo Agropec (Cañete - Lima) M. owari $\mathrm{x}$ M. king y Citrus unshiu var. owari 3.III.5 (3), Fundo Monserrat (Irrigación Santa Rosa - Lima) Citrus sinensis (L.) var. washington navel 21.III.5 (5), Fundo La Tapera (Huaral - Lima): Citrus unshiu var. owari 15.IV.5, 18.IV.5, 20.IV.5 (5).

Observaciones: Las medidas de 22 hembras adultas colectadas en este trabajo son semejantes a la descripción de El-Banhawy (1979) con especimenes de Santa Rosa, Departamento de Lima (Perú). Los promedios y el intervalo (en paréntesis) de las medidas del cuerpo en $\mu \mathrm{m}$ son: la placa dorsal 359 (336-390) de largo y 168 (160-175) de ancho; j1 23 (20-25), j3 34 (31-38), j4 25 (21-32), j5 25 (21-32), j6 33 (25-34), J2 36 (28-46), J5 13 (10-15), z2 33 (2935), z4 35 (28-40), z5 26 (21-33), Z1 38 (33-40), Z4 54 (48-62), Z5 64 (65-74), s4 43 (38-50), S2 46 (4053), S4 42 (33-48), S5 35 (30-43), r3 28 (18-32), R1 26 (21-30); St IV 51 (48-55); St1-St3 62 (60-65), St2St2 58 (55-65), St5-St5 69 (57-65), la placa ventrianal 122 (113-135) de largo y 87 (83-95) de ancho; cáliz de la espermateca 11 (10-15) de largo. Peritrema presente entre $\mathrm{j} 1 \mathrm{y} \mathrm{j} 3$.

Se observó a Neoseiulus californicus depredando huevos de $P$. citri, tanto en laboratorio como en campo. Esta especie ha sido encontrada también en Ricinus communis en Chincha.

\section{Subfamilia: TYPHLODROMINAE Scheuten 1857 Género: Typhlodromus Scheuten 1857 Subgénero: Anthoseius De Leon 1968}

\section{Typhlodromus (Anthoseius) evectus Schuster 1966} (Figura 4)

Material examinado: Fundo El Paraíso (Irrigación Santa Rosa - Lima): Citrus sinensis var. washington Navel 24.X.3 (3), Fundo Hoja Redonda (Chincha Ica) Citrus unshiu var. owari (1) 3.XI.3, Fundo Ramos (Cañete - Lima): M. owari x M. king y M. clementina x M. dancy (1) 27.XI.3, 8.I.4, 4.III.4, 1.IX.4, 31.III.5, 2.II.5, 7.IV.5, Fundo San Martín (Cañete - Lima): Citrus clementina 28.XI.3 (1), Fundo Tranquera Blanca (Cañete - Lima): M. clementina x M. dancy 23.XII.3, Fundo La Calera (Chincha - Ica): M. king x M. dancy 28.I.4 (5), Fundo Luis Calle (Irrigación Santa Rosa - Lima): Mandarina Citrus reticultata 15.II.5 (1), Fundo Monserrat (Irrigación Santa Rosa Lima) Citrus sinensis var. washington navel 21.III.5 (3), 4.IV.5, 7.IV.5, Fundo La Tapera (Huaral - Lima): Citrus unshiu var. owari 15.IV.5, 18.IV.5, 20.IV.5 (3).

Observaciones: Las medidas de 22 hembras adultas colectadas en este trabajo son semejantes a la descripción original (Schuster, 1966). Los promedios y el intervalo (en paréntesis) de las medidas del cuerpo en $\mu \mathrm{m}$ son: la placa dorsal 307 (290-320) de largo y 171 (165-185) de ancho; j1 22 (20-23), j3 27 (22-30), j4 20 (17-25), j5 20 (19-22), j6 27 (25-29), J2 30 (2735), J5 10 (10-11), z2 21 (20-23), z3 27 (25-30), z4 29 (28-34), z5 23 (20-25), Z4 45 (43-48), Z5 53 (47-59), s4 31 (29-34), s6 32 (31-36), S2 36 (33-40), S4 36 (33-38), S5 21 (17-25), r3 28 (27-30), R1 26 (25-28); St IV 25 (22-28); St1-St3 58 (59-61), St2-St2 54 (4561), St5-St5 53 (48-54), la placa ventrianal 102 (98105) de largo y 65 (63-70) de ancho. Cáliz de la espermateca 17 (13-20) de largo. Peritrema presente en la base de la j1.

Se observó a $T$ (A). evectus alimentándose de huevos, larvas, protoninfas, deutoninfas y adultos de $P$. citri. Fue observado que colocaban los huevos aglomeradamente. T. evectus fue encontrado en plantas de cítricos sin arañitas rojas, pero también en plantas con alto grado de infestación de arañitas rojas (más de 50 P. citri por hoja). En el fundo Monserrat (Santa Rosa) esta especie fue encontrada ampliamente dispersa, excepto en sectores que habían sido lavados 
o aplicados con pesticidas. Primer registro para el Perú

\section{Typhlodromus (Anthoseius) transvaalensis Nesbitt 1951 \\ (Figura 5)}

Material examinado: Fundo La Venturosa (Nazca Ica) Citrus sinensis var. valencia 20.II.5 (1).

Observaciones: La medida de una hembra adulta colectada en este trabajo es semejante a la descripción de Schicha (1981), excepto que la seta S5 es aproximadamente un $40 \%$ más pequeña, además el escudo ventrianal (EVA) es 13\% más angosto y $17 \%$ más corto. Las medidas del cuerpo en $\mu \mathrm{m}$ son: la placa dorsal 345 de largo y 175 de ancho; j1 30, j3 36, j4 29, j5 30, j6 34, J2 44, J5 7, z2 21, z3 41, z4 37, z5 27, Z4 52, Z5 60, s4 42, s6 46, S2 52, S4 47, S5 5, r3 33, R1 40; Sge IV 39, Sti IV 22, St IV 50; St1-St3 67, St2St2 63, St5-St5 74, la placa ventrianal 92 de largo y 73 de ancho; cáliz de la espermateca 26 de largo. Peritrema presente entre j3 y z2. Primer registro para el Perú

\section{Género: Typhlodromina Muma 1961 Typhlodromina subtropica Muma \& Denmark (Figura 6)}

Material examinado: Fundo Ramos (Cañete - Lima): M. owari x M. king 27.XI.3, 2.II.5, 7.IV.5 (4).

Observaciones: Las medidas de 4 hembras adultas colectadas en este trabajo son semejantes a la descripción de Chant \& Yoshida-Shaul (1983), excepto que las setas j3, z2, z3, z5 y Z5 no se pudieron comparar porque en la descripción de Chant \& Yoshida-Shaul (1983), dichas setas estaban rotas. Además los especimenes colectados son ligeramente más grandes. Los promedios y el intervalo (en paréntesis) de las medidas del cuerpo en $\mu \mathrm{m}$ son: la placa dorsal 376 (370-385) de largo y 256 (255-260) de ancho; j1 30 (28-32), j3 47 (46-47), j4 41 (39-45), j5 44 (41-48), j6 56 (54-61), J2 71 (63-71), J5 12 (1213), z2 40 (35-43), z3 51 (44-60), z4 57 (53-63), z5 45 (42-48), Z4 71 (70-73), Z5 59 (55-62), s4 66 (58-62), s6 71 (63-77), S5 70 (67-73), r3 37 (33-41), R1 29 (22-33); la placa ventrianal 120 (110-125) de largo y 80 (80-81) de ancho; cáliz de la espermateca 24 (2232) de largo. Esta especie ha sido encontrada también en Vitis vinifera en Sunanpe - Chincha.

\section{I.2. Familia Cheyletidae Leach}

\section{Mexecheles sp.}

Material examinado: Fundo Calle (Irrigación Santa Rosa - Lima): Citrus reticultata 15.II.5 (1).

\section{Cheletogenes sp.}

Material examinado: Fundo Luis Calle (Irrigación Santa Rosa-Lima): Citrus reticultata 15.II.5 (1).

\section{I.3. Familia Cunaxidae Thor}

\section{Armascirus sp.}

Material examinado: Fundo Ramos (Cañete- Lima): M. owari x M. king 27.XI.3 (2).

\section{Insectos predadores de $P$. citri \\ II.1. Orden Coleoptera}

\section{Stethorus Weise 1885 \\ Stethorus tridens Gordon 1982}

Material examinado: Fundo Anglia (Nazca - Ica) Citrus sinensis (L.) var. washington navel. 20. II.5 (14), Fundo La Venturosa (Nazca - Ica) Citrus sinensis (L.) var. valencia late selección Limera 20.II.05 (17), Fundo Ramos (Cañete- Lima): M. owari x M. king 7.IV.5 (3), Fundo Monserrat (Irrigación Santa Rosa-Lima) Citrus sinensis (L.) var. washington navel 21.III.5 (20), Fundo La Tapera (Huaral - Lima) Citrus unshiu var. owari 15.IV.5, 18.IV.05, 20.IV.5 (5).

Observaciones: $S$. tridens se encontraba depredando sólo en plantas que presentaban alta infestación de $P$. citri (más de 50 P. citri por hoja). En estos campos, se observaron posturas, larvas y adultos de $S$. tridens, en tanto que en lugares donde no habia alimento, no fue encontrado. $S$. tridens fue encontrado generalmente en parejas.

\section{Stethorus histrio Chazeau}

Material examinado: Fundo Anglia (Nazca - Ica) Citrus sinensis (L.) var. washington navel. 20. II.5 (5), Fundo La Venturosa (Nazca - Ica) Citrus sinensis (L.) var. valencia late selección Limera 20.II.5 (15).

\section{Oligota sp.}

Material examinado: Fundo Monserrat (Irrigación Santa Rosa - Lima) Citrus sinensis (L.) var. washington navel 21.III.5 (1).

\section{II.2. Orden Neuroptera}

\section{Chrysoperla externa Hagen 1861}

Material examinado: Fundo Ramos (Cañete - Lima): M. owari x M. king y M. clementina x M. dancy (4) 27.XI.3, 8.I.4, 4.III.4, 1.IX.4, 31.III.05, 2.II.5, 07.IV.5, Fundo Monserrat (Irrigación Santa Rosa - Lima) Citrus sinensis var. washington navel 21.III.5 (3), 
Fundo La Tapera (Huaral - Lima): Citrus unshiu var. owari 15.IV.5, 18.IV.5, 20.IV.5 (2).

\section{Ceraeochrysa cincta Schneider 1851}

Material examinado: Fundo Ramos (Cañete - Lima): M. owari x M. king y M. clementina x M. dancy (2) 27.XI.3, 8.I.04, 4.III.4, 1.IX.4, 31.III.5, 2.II.5, 7.IV.5.

\section{I.3. Orden Thysanoptera} Aeolothrips sp.

Material examinado: Fundo Ramos (Cañete - Lima): M. owari x M. king y M. clementina x M. dancy (2) 27.XI.3.

\section{Discusión}

Predadores asociados a $P$. citri en la costa central del Perú

La baja riqueza de especies de predadores asociadas a $P$. citri durante las colectas en la costa central del Perú probablemente sea debida al clima de desierto costero de todas las áreas muestreadas, y/o porque los muestreos fueron realizados en fundos de cítricos con aplicaciones químicas periódicas.

A. chungas fue predominante en Huaral y Cañete, $N$. californicus predominó en Chincha. T. evectus predominó en La Irrigación Santa Rosa, este último se caracteriza por ser un ambiente con humedad relativa más baja que las otras localidades.

Los insectos predadores Stethorus tridens y Stethorus histrio fueron encontrados asociados a P.citri principalmente en Nazca.

¿Cumplen un rol importante los predadores asociados a $P$. citri en la costa central del Perú?

De acuerdo con las observaciones en campo, se puede afirmar la existencia de un control natural de $P$. citri, lo que probablemente ocasiona que la plaga no llegue a niveles altos en algunas zonas. En hojas de plantas con niveles medios de infestación de $P$. citri fue comprobada la presencia de A. chungas, $N$. californicus y $T$. evectus en la mayoría de las observaciones. De otro lado hojas infestadas con P.citri sin predadores asociados alcanzaban generalmente niveles altos de infestación de $P$. citri. Por tanto, y aunque la predación natural en campo muchas veces no llega a ser capaz de bajar la población de $P$. citri a niveles que no dañen al cultivo, pero llegan a retardar su crecimiento poblacional, este hecho hace imprescindible el mantenimiento de poblaciones de ácaros predadores en las plantas cítricas en la costa central del Perú, para evitar que el ataque de los $P$. citri sea más severo de lo que ya lo es actualmente.

A. chungas es un ácaro endémico de Perú, fue encontrado por primera vez en Motupe (Denmark \&
Muma, 1989). Las especies del género Amblyseius, al cual pertenece $A$. chungas, son consideradas como predadoras generalistas de acuerdo con McMurtry \& Croft (1997); es decir, se alimentan ácaros, estadios inmaduros de insectos y hasta polen.

Neoseiulus californicus, otro ácaro predador frecuente en los muestreos, se distribuye en África, América, Asia y Europa (Moraes et al., 2004). Además de ser reconocido como controlador biológico de $P$. citri en varias partes del Mundo (McMurtry., 1977; García-Marí et al., 1986; Copping, 2001), según McMurtry \& Croft (1997) la depredación de esta especie es considerada del tipo II, es decir, se alimentan de ácaros de la familia Tetranychidae que producen poca tela, como por ejemplo $P$. citri y, ocasionalmente de polen o de exudaciones de plantas. La población de $N$. californicus presente en la costa central del Perú son de mayor tamaño corporal que el de otras poblaciones en América (El-Banhawy, 1979).

Las especies del género Typhlodromus, al cual pertenece $T$. evectus, son consideradas por McMurtry \& Croft (1997) como predadores generalistas.

Es deseable, a partir de todas estas informaciones adquiridas en este trabajo, una investigación más profunda de los parámetros biológicos y el comportamiento predador en laboratorio y campo de A. chungas, $N$. californicus y T. evectus.

Typhlodromus transvaalensis, Typhlodromina subtropica y Phytoseiulus persimilis son ácaros de la familia Phytoseiidae que por su poca frecuencia no se les considera importantes controladores de $P$. citri. No obstante, la especie $P$. persimilis, es la especie de ácaro predador más usada a nivel mundial en el control biológico del ácaro plaga cosmopolita Tetranychus urticae (Gerson et al., 2003).

Mexecheles sp. Cheletogenes sp. y Armascirus sp. son ácaros de otras familias predadoras, pero sin grande potencial debido a su rareza en el campo en asociación a $P$. citri.

El papel regulador de las mariquitas predadoras de ácaros (Stethorus spp.) es probablemente diferente al de los ácaros predadores en campo. Según Doreste (1988), larvas y adultos de éstas se alimentan de huevos, juveniles y adultos de tetraníquidos. Se ha determinado que los Stethorus adultos se alimentan aproximadamente de 40 arañitas por día, mientras que durante el período de larva pueden consumir más de 200 arañitas. De acuerdo con los estudios de Hull (1995) en Pensilvania, los adultos de Stethorus punctum pueden llegar a consumir de 75 a 100 arañitas (Panonychus ulmi) por día y las larvas más de 75 arañitas en el mismo lapso de tiempo; con lo que es 
posible disminuir rápidamente un ataque violento de estos ácaros. Una desventaja de usar mariquitas en el control de $P$. citri es el hecho de que éstas generalmente no ovipositan cuando las arañitas están en grado bajo de infestación, y sólo lo hacen cuando $P$. citri se encuentran en un grado de infestación alto.

Oligota sp., Chrysoperla externa, Ceraeochrysa cincta y Aeolothrips sp. son insectos predadores, pero raras veces encontrados asociados con $P$. citri.

\section{Agradecimientos}

$\mathrm{Al} \mathrm{PhD.} \mathrm{Gilberto} \mathrm{José} \mathrm{de} \mathrm{Moraes} \mathrm{(Universidad} \mathrm{de}$ Sao Paulo - Brasil) por la orientación y confirmación de las identificaciones de las especies de ácaros de la familia Phytoseiidae.

A PROCITRUS por la ayuda financiera para la realización del presente trabajo.

Al Museo de Entomología de la Universidad Agraria La Molina, por haber dado algunos de los especimenes identificados en este estudio.

\section{Literatura citada}

Aguilar F., Salazar J. \& Núñez E. 1980. El cultivo de Cítricos. Rev. Per. Ent. 23 (1): 97-102.

Beingolea O. 1959. El problema de la Mosca Blanca Lanuda de los Cítricos. Aleurothrixus floccosus Mask, en el Perú. Rev. Per. Ent. Agr. 2 (1): 65-68

Beingolea O., Salazar J. \& Murat I. 1968. Control Integrado de Plagas de Cítricos en el Fundo Santa Teresa (Sunampe, Chincha): Proyecto III, Centro de Introducción y Cría de Insectos Útiles. Informes de Circulación interna Sub-Dirección de Sanidad Vegetal Nos. 1-2-3-4-5-7 y N8-68-SubD/SV.

Beingolea O., Salazar J. \& Murat I. 1969. La rehabilitación de un huerto de cítricos, como ejemplo de la factibilidad de aplicar sistemas de control de las plagas de los cítricos en el Perú. Rev. Per. Ent. 12 (1): 3-45.

Boyne J.V. \& Hain F..P. 1983. Response of Neoseiulus fallacis (Acarina: Phytoseiidae) to different prey densities of Oligonychus ununguis (Acarina: Tetranychidae) and to different relative humidity regimes. Can. Entomol. 115: 1607-14.

Chant D.A. \& Yoshida-Shaul E. 1983. A world review of five similar species groups in the genus Typhlodromus Scheuten: Part II. The conspicuus and cornus groups (Acarina: Phytoseiidae). Canadian Journal of Zoology, Canada. 61: 1041-1057.

Copping L.G. (Ed) 2001. The BioPesticide Manual. $2^{\text {nd }}$ edn. British Crop Protection Council, Surrey.

De Moraes G.J. \& McMurtry J.A. 2005. Key to the genera of Phytoseiidae (Adult Females). Working draft, not for publication.

Denmark H.A. \& Muma M.H. 1989. A revision of the genus Amblyseius Berlese, 1914 (Acari: Phytoseiidae). Occasional Papers of the Florida State Collection of Arthropods, USA, 4.

Denmark H.A. \& Schicha E. 1983. Revision of the genus Phytoseiulus Evans (Acarina: Phytoseiidae). International Journal of Acarology, USA. 9: 27-35.
Doreste E. 1988. Acarología. Instituto Interamericano de Cooperación para la Agricultura (IICA). San José, Costa Rica.

El-Banhawy E.M. 1979. Records on phytoseiid (Acari) mites of Peru. International Journal of Acarology, USA. 5(2): 111-116.

García-Marí F., Ferragut F., Marzal C., Costa-Cornelles J., \& Loborda R. 1986. Ácaros que viven en las hojas de los cítricos españoles. Lev. Agr. Prod. Prot Vegetal. 1 (2): 61-92.

Gerson U., Smiley R. \& Ochoa R. 2003. Mites (Acari) for Pest Control Backwell Science. First Edition. : 103-113 .

Gordon R.D. 1982. New species and new synonymy in neotropical Stethorus Weise (Coleoptera). Coleopterists Bull. 36: 121-126.

Gordon, R. D. \& Chapin, E. A. 1983. A Revision of the new world species of Stethorus Weise (Coleoptera: Coccinellidae). Transactions of the American Entomological Society. 109 (3): 229-276.

Hull L. 1995. Know Your Friends: Stethorus punctum. Midwest Biological Control News Online. 2 (12). www.nysaes.cornell.edu.

Krantz G.W. 1978. A manual of acarology. Second Edition. Editor Oregon State University Book Store, Inc. Oregon University. Printed in The United States.

Ludwig J.A. \& Reynolds J.F. 1988. Statistical ecology: A primer on methods and computing. New York: Wiley.

McMurtry J.A. 1977. Biological control of citrus mites. En: W.Grierson (Editor), Proc. Int. Soc. Citriculture. 2. Painter Printing, De León Springs. : 456-459

McMurtry J.A. \& Croft B.A. 1997. Life styles of phytoseiid mites and their roles in biological control. Annu. Rev. Entomol. 42: 291-321.

McMurtry J.A. \& Moraes G.J. de 1989. Some phytoseiid mites from Peru with descriptions of four new species (Acari: Phytoseiidae). International Journal of Acarology, USA. 15(3): 179-188.

Moraes, G.J. de, McMurtry, J.A., Denmark, H.A. \& Campos, C.B. 2004. A revised catalog of the mite family Phytoseiidae. Zootaxa, 434, 1 - 494.

Mori H. \& Chant D.A. 1966. The influence of humidity on the activity of Phytoseiulus persimilis Athias-Henriot and its prey. Tetranychus urticae (C. L. Koch) (Acarina: Phytoseiidae, Tetranychydae). Can. J. Zool. 44: 863-71.

Navarrete-Heredia J.L., Newton A.F., Thayler M.K., Ashe J.S. \& Chandler D.S. 2002. Guía ilustrada para los géneros de Staphylinidae de México. Universidad de Guadalajara. Comisión Nacional para el Conocimiento y Uso de la Biodiversidad (CONABIO).

Núñez E. 1988. Chrysopidae (Neuroptera) del Perú y sus especies más comunes. Rev. per. Ent. 31: 69-75.

Odum E.P. 1988. Ecologia. Rio de Janeiro: Guanabara.

Pritchard A.E. \& Baker E.W. 1955. A Revision of the Spider Mite Family Tetranychidae. Pac. Coast Entom. Soc. Mem. Ser. 2: 1-472.

ProCitrus, 2005. Investigación: Avances del proyecto de control biológico PROCITRUS - SENASA. Citrinotas. Boletín informativo de PROCITRUS. 27: 8.

Reuveny H., Palevsky E. \& Gerson U. 1996. Laboratory life history studies of the predaceous mite Typhlodromus athiasae (Acarina: Phytoseiidae). Syst. Appl. Acarol. 1: 45-53.

Schicha E. 1981. Two new species of Amblyseius Berlese from Queensland and New Caledonia compared with $A$. 
largoensis (Muma) from the South Pacific and $A$. deleoni Muma and Denmark from New South Wales (Acari : Phytoseiidae). J. Australian Entomol. Soc. 20: 101-109.

Schuster R.O. 1966. Phytoseiidae of the Galapagos Islands (Acarina: Mesostigmata). Pacific Insects, Hawaii. 8: 319-339.

Simpson E.H. 1949. Measurement of diversity. Nature, 163.
Stehr F.W. 1991. Immature insects. Vol. 2. Kendall/Hunt, Dubuque, Iowa.

Yauri E. 2001. Colección y caracterización de controladores biológicos en frutales. Informe interno Anual 2001 E. E. Donoso (INIA) - Huaral. Programa Nacional de Investigación en Manejo Integrado de Plagas Informe de Experimento Concluido. Formato DGIA-041.

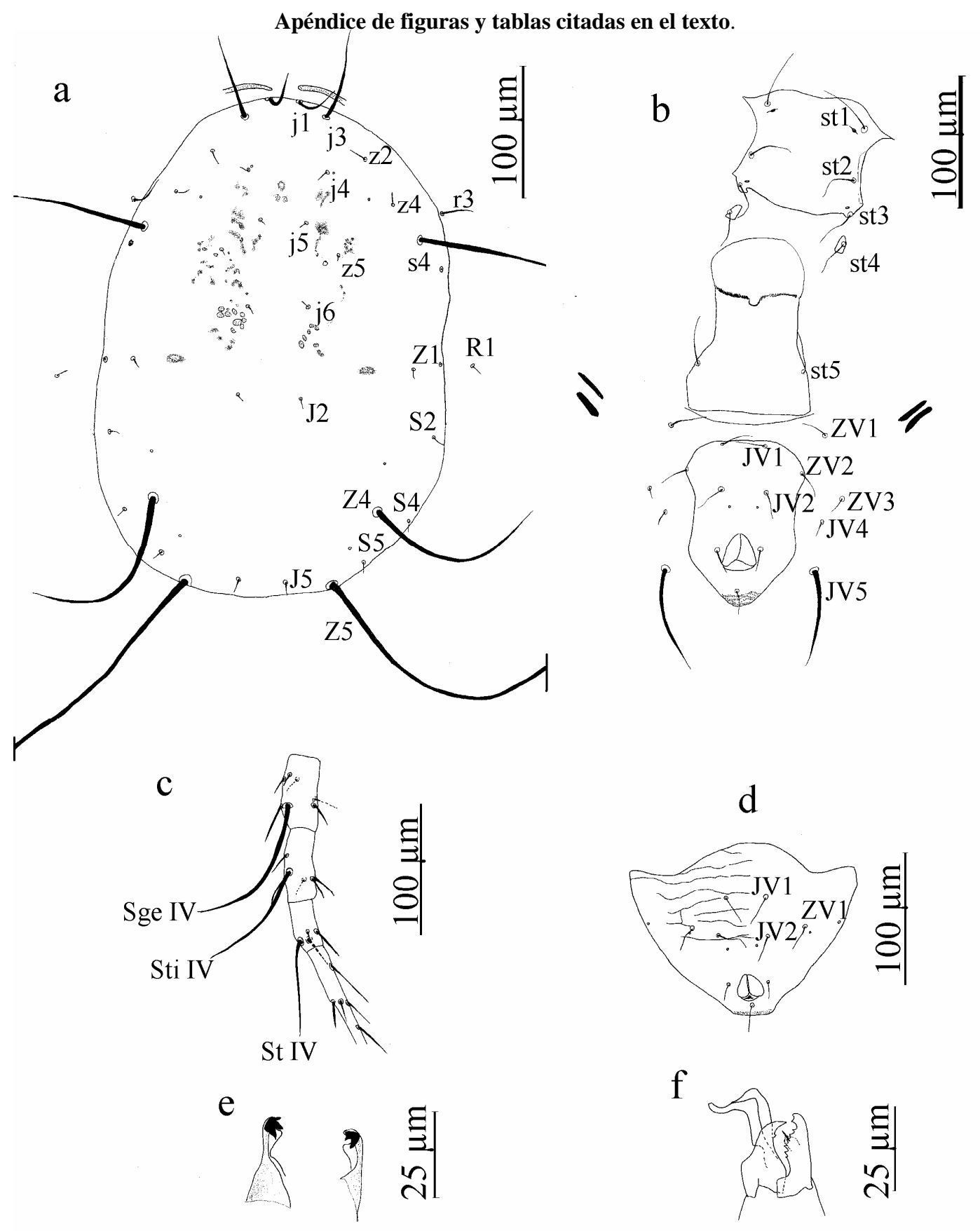

Figura 1. Amblyseius chungas. a. Placa dorsal de hembra, b. Placas ventrales de hembra, c. Pata IV de hembra, d: Placa ventrianal de macho, e: Variaciones de la espermateca, f: Espermidáctilo (macho). 

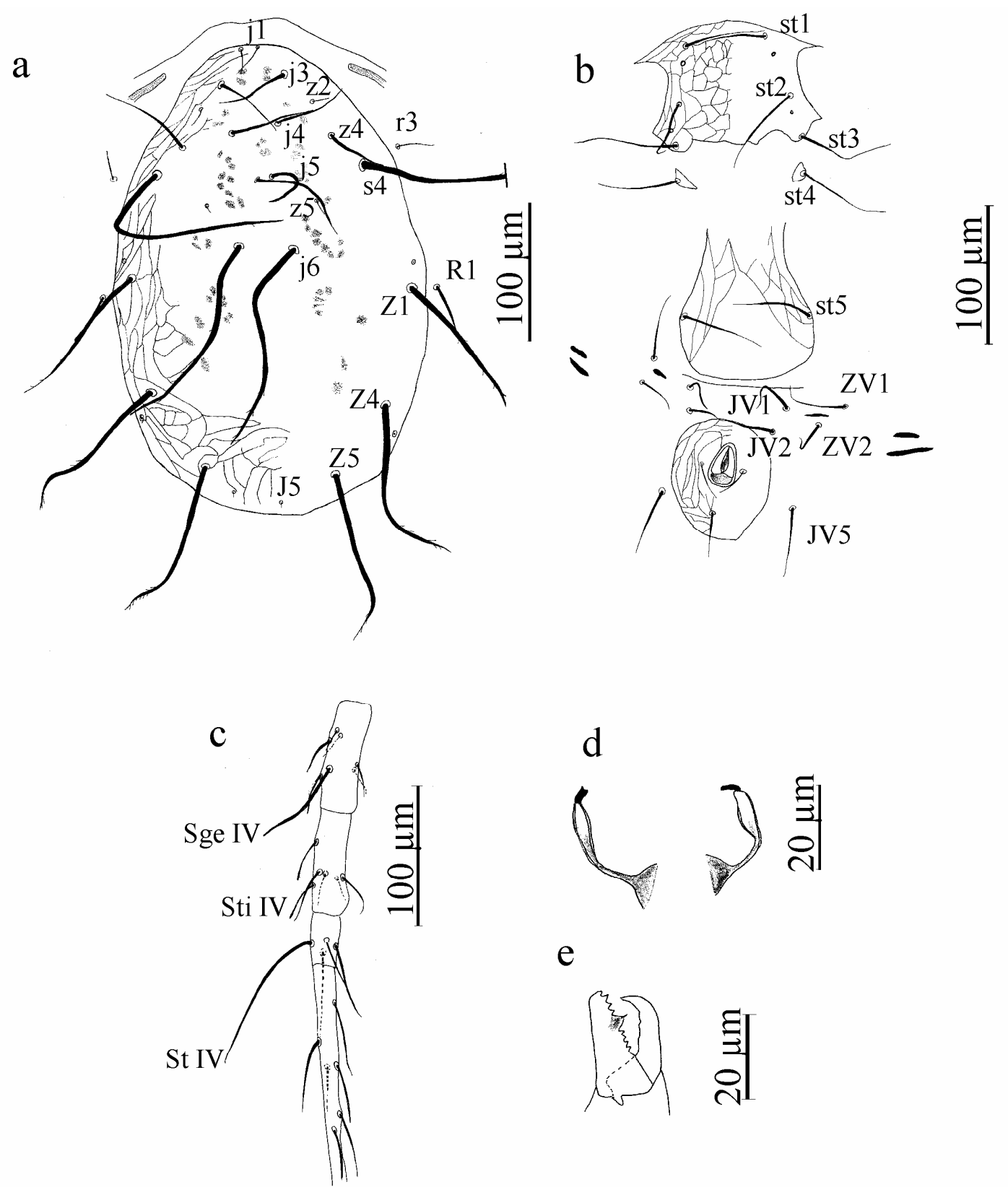

e

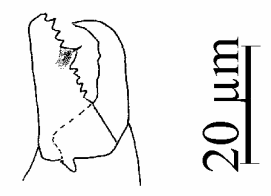

Figura 2. Phytoseiulus persimilis. a. Placa dorsal de hembra, b. Placas ventrales de hembra, c. Pata IV de hembra, d: Variaciones de la espermateca, e: Quelícera (hembra). 

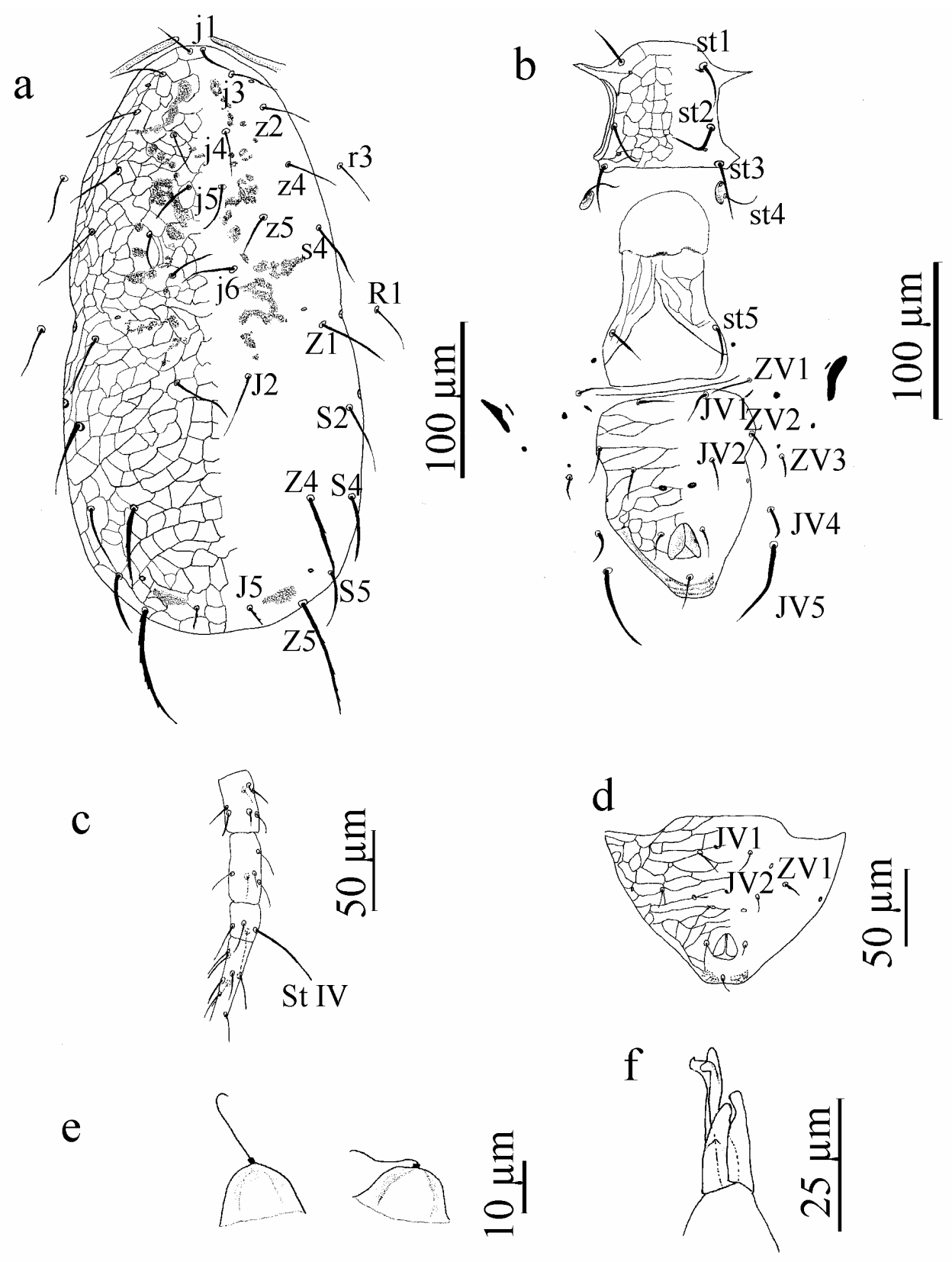

Figura 3. Neoseiulus californicus. a. Placa dorsal de hembra, b. Placas ventrales de hembra, c. Pata IV de hembra, d: Placa ventrianal de macho, e: Variaciones de la espermateca, f: Espermidáctilo (macho). 

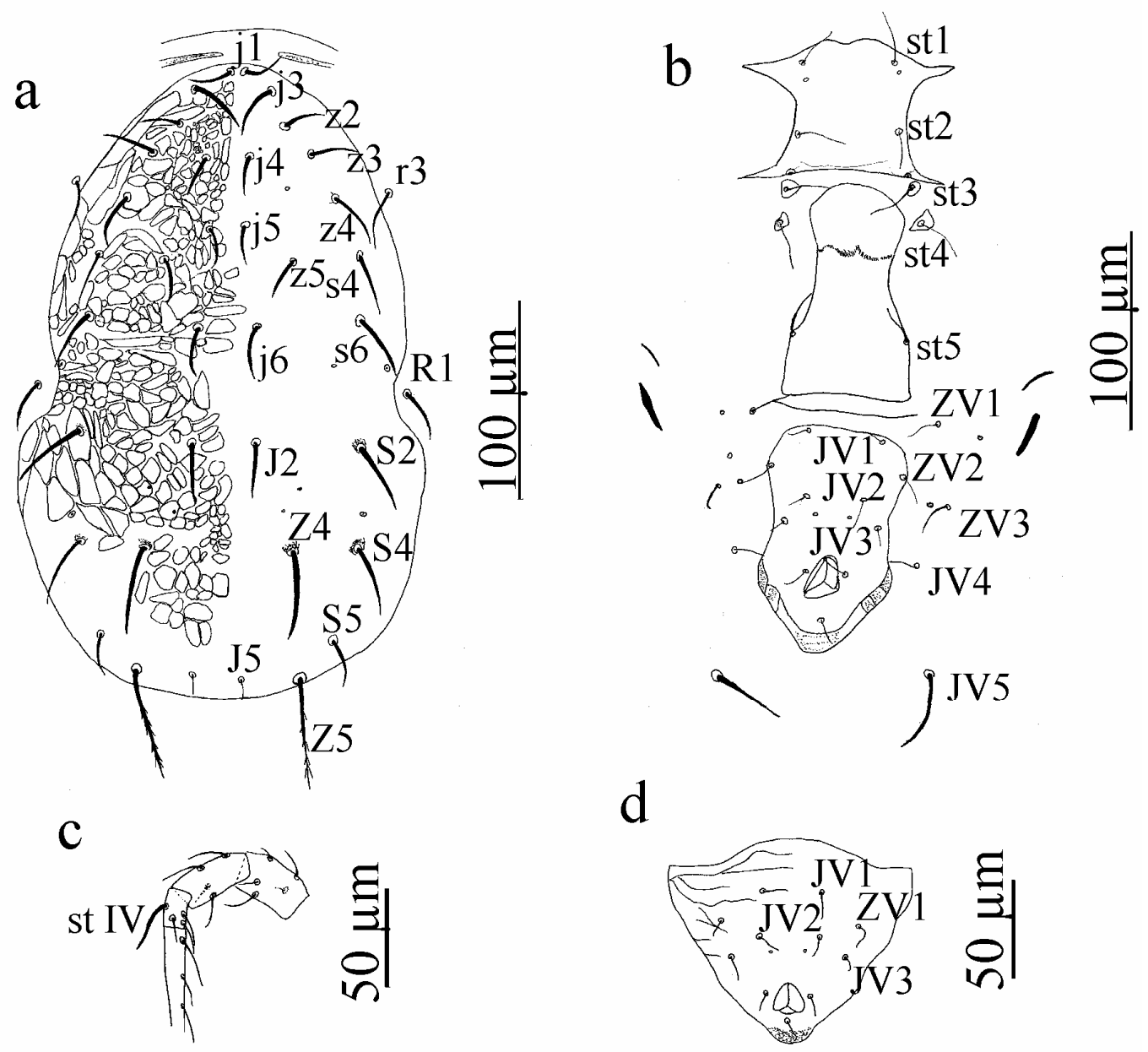

d
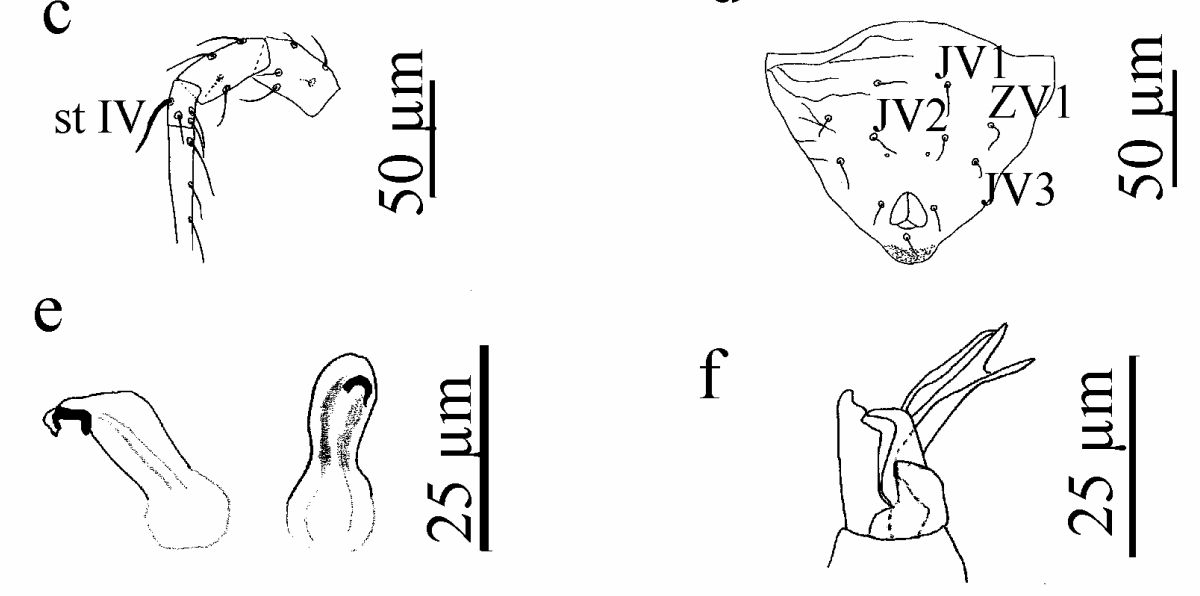

Figura 4. Typhlodromus evectus. a. Placa dorsal de hembra, b. Placas ventrales de hembra, c. Pata IV de hembra, d: Placa ventrianal de macho, e: Variaciones de la espermateca, f: Espermidáctilo (macho). 


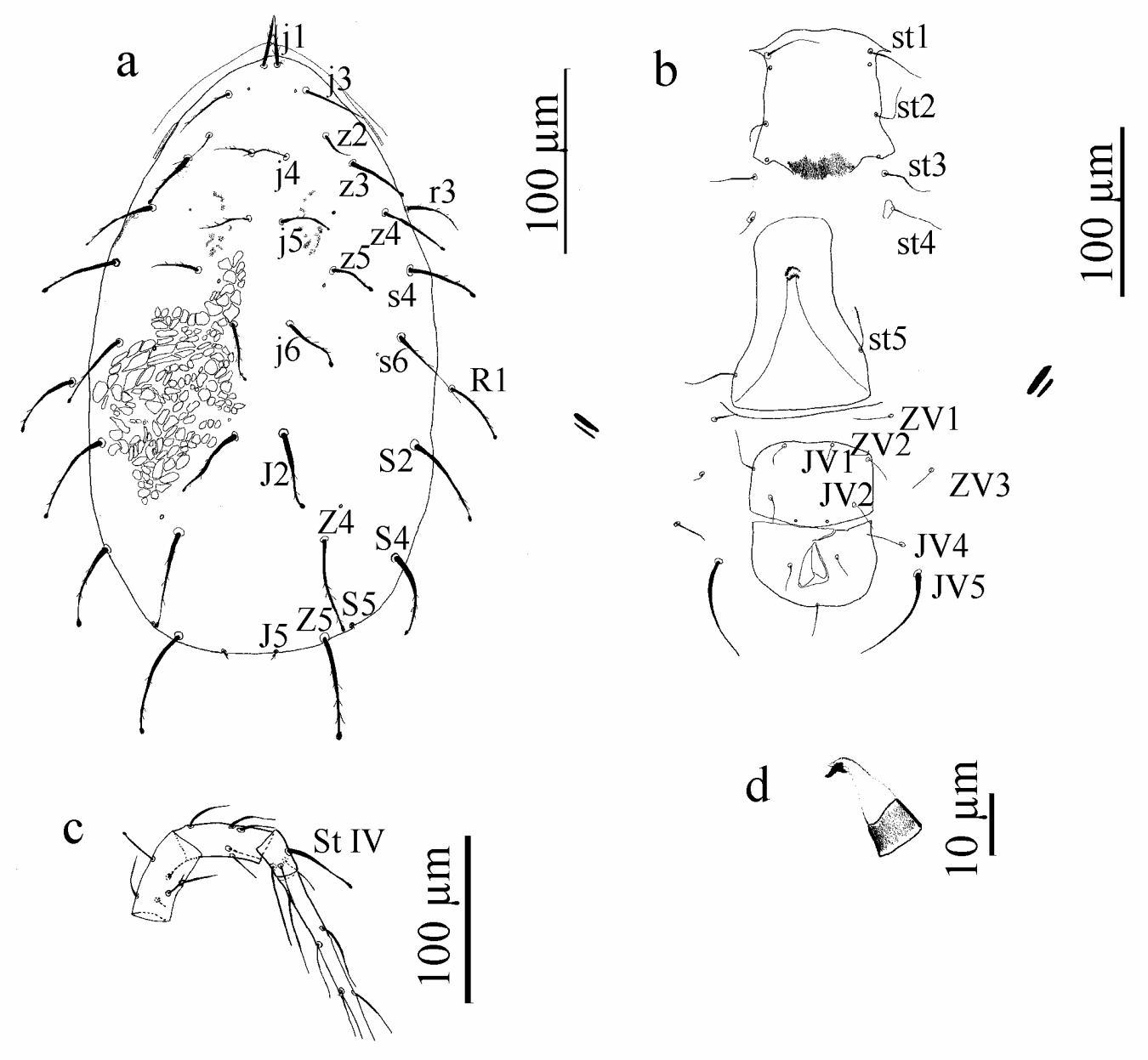

Figura 5. Typhlodromus transvaalensis. a. Placa dorsal de hembra, b. Placas ventrales de hembra, c. Pata IV de hembra, d: Espermateca. 

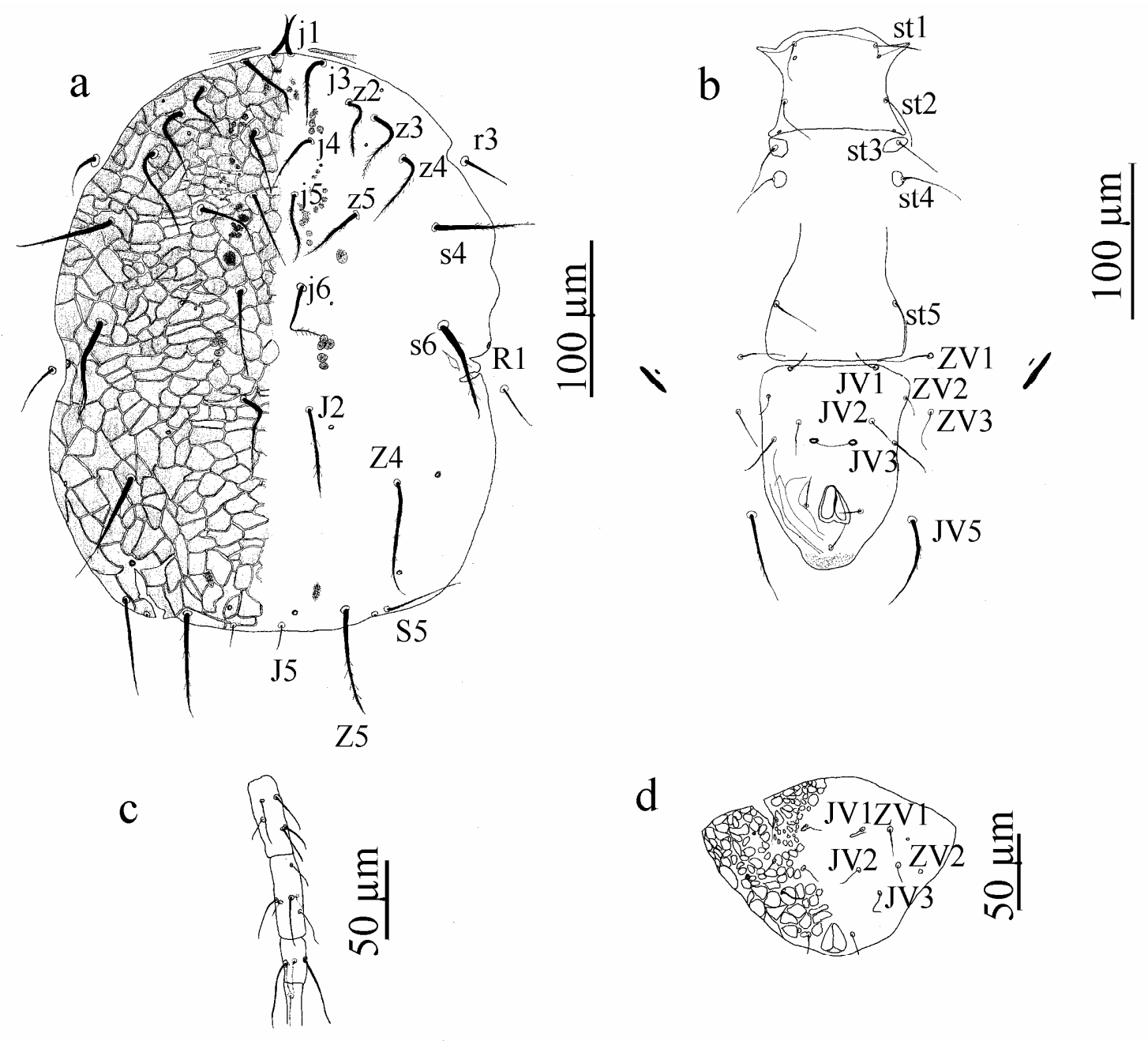

d
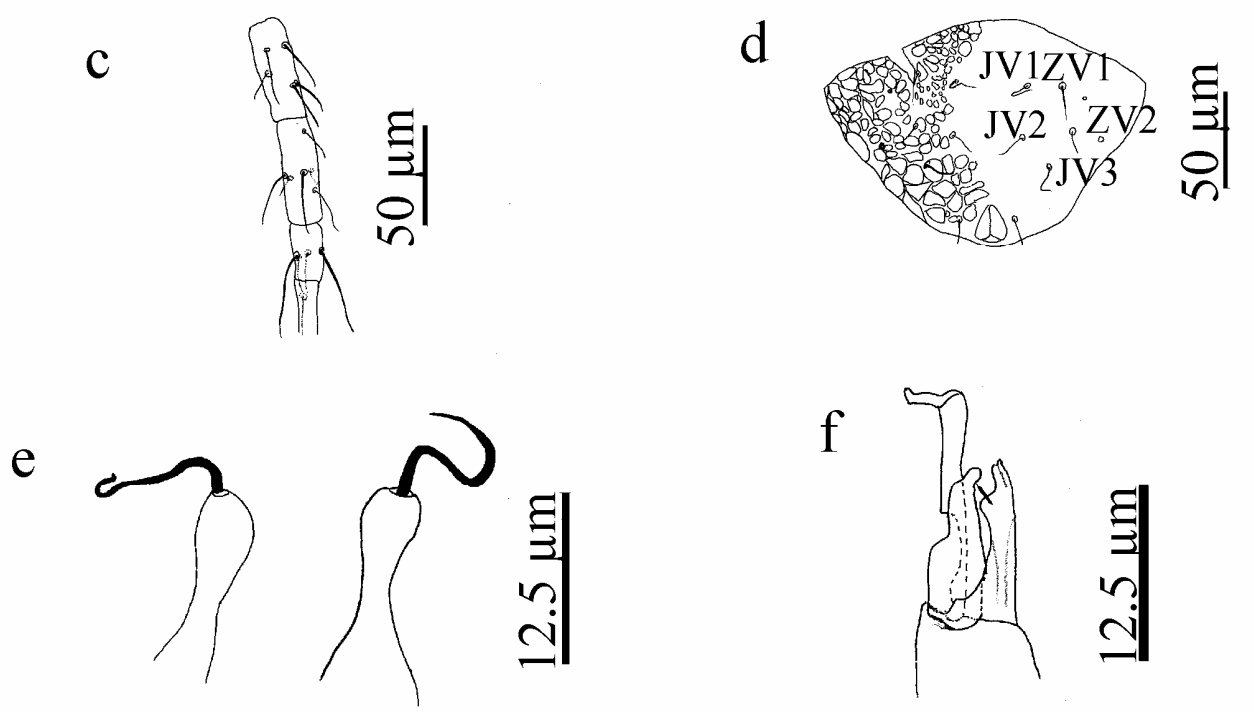

Figura 6. Typhlodromina subtropica. a. Placa dorsal de hembra, b. Placas ventrales de hembra, c. Pata IV de la hembra, d: Placa ventrianal de macho, e: Variaciones de la espermateca, f: Espermidáctilo (macho). 
Tabla 1. Predadores asociados a P. citri en la áreas colectadas de colecta de la costa central del Perú.

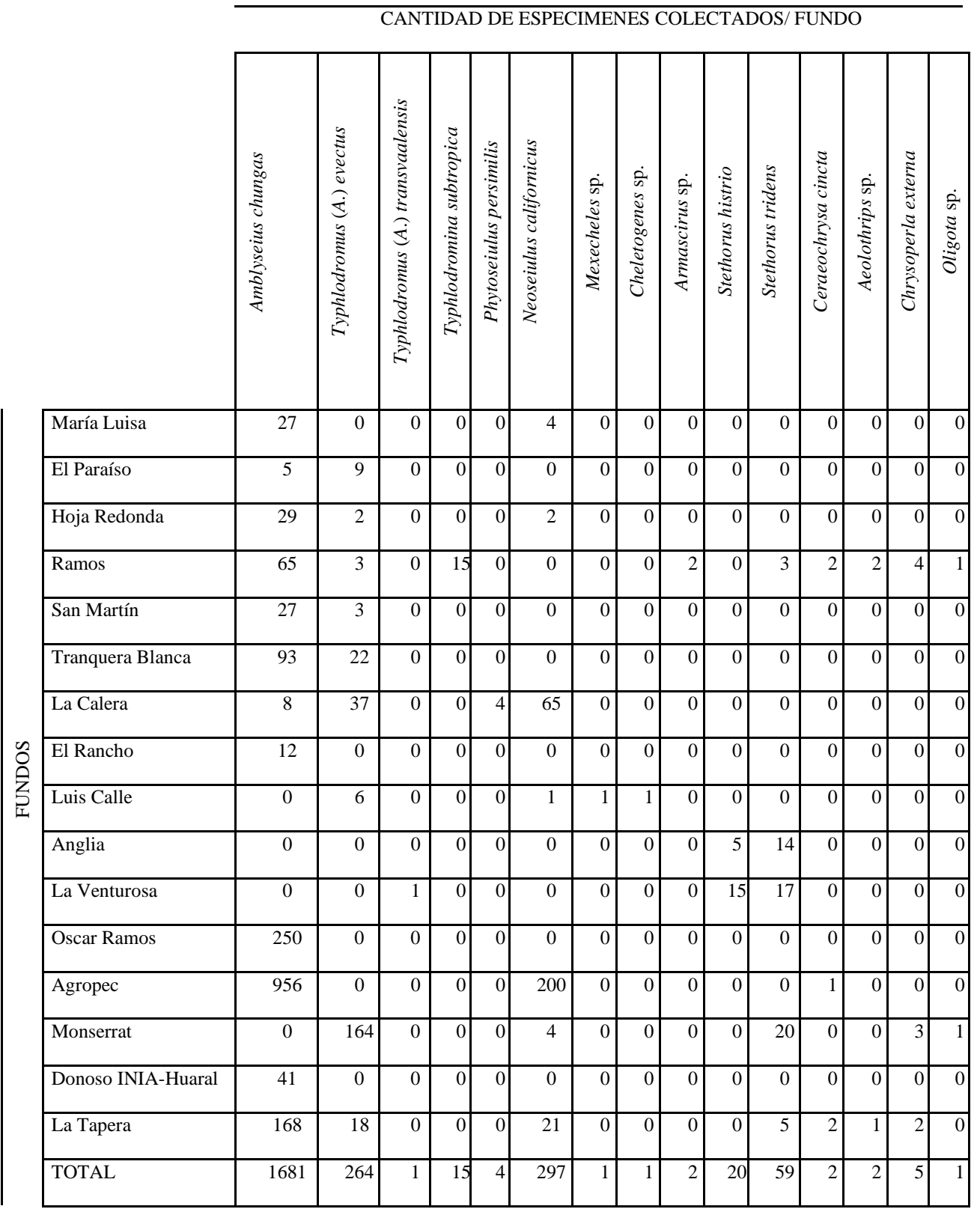

\footnotetext{
${ }^{1}$ Afiliación institucional en el momento de la investigación: Facultad de Ciencias Biológicas Universidad Nacional Mayor de San Marcos Av. Venezuela Cdra. 34 s/n Lima 01, Peru. Afiliación institucional en el momento de la publicación: Departamento de Entomologia, Fitopatologia e Zoologia Agrícola ESALQ/USP 13418-900 Piracicaba - SP, Brasil. a_guanilo@yahoo.co.uk

${ }^{2}$ Facultad de Ciencias Biológicas Universidad Nacional Mayor de San Marcos. nmartinezl@unmsm.edu.pe
} 